\title{
Development of an Indirect ELISA to Detect Equine Antibodies to Theileria haneyi
}

\author{
Reginaldo G. Bastos ${ }^{1}$, Kelly P. Sears ${ }^{1}$, Kelcey D. Dinkel ${ }^{1}$, Lowell Kappmeyer ${ }^{2} \mathbb{D}$, Massaro W. Ueti ${ }^{1,2}$, \\ Donald P. Knowles ${ }^{1}$ and Lindsay M. Fry ${ }^{1,2, *}$
}

1 Department of Veterinary Microbiology \& Pathology, College of Veterinary Medicine, Washington State University, Pullman, WA 99164, USA; reginaldo_bastos@wsu.edu (R.G.B.); kellyp.sears@wsu.edu (K.P.S.); kelcd23@hotmail.com (K.D.D.); massaro.ueti@usda.gov (M.W.U.); dknowles@wsu.edu (D.P.K.)

2 Animal Disease Research Unit, USDA-ARS, Pullman, WA 99164, USA; Lowell.kappmeyer@usda.gov

* Correspondence: Lindsay.fry@usda.gov

Citation: Bastos, R.G.; Sears, K.P.; Dinkel, K.D.; Kappmeyer, L.; Ueti, M.W.; Knowles, D.P.; Fry, L.M. Development of an Indirect ELISA to Detect Equine Antibodies to Theileria haneyi. Pathogens 2021, 10, 270. https://doi.org/10.3390/ pathogens 10030270

Academic Editor: Siddhartha Das

Received: 29 January 2021

Accepted: 23 February 2021

Published: 27 February 2021

Publisher's Note: MDPI stays neutral with regard to jurisdictional claims in published maps and institutional affiliations.

Copyright: (c) 2021 by the authors. Licensee MDPI, Basel, Switzerland. This article is an open access article distributed under the terms and conditions of the Creative Commons Attribution (CC BY) license (https:/ / creativecommons.org/licenses/by/ $4.0 /)$.

\begin{abstract}
The apicomplexan parasite Theileria haneyi is one of two known causative agents of equine theileriosis. It causes milder clinical disease than its more virulent counterpart, Theileria equi, in experimentally infected horses, and can superinfect T. equi-positive horses. The current equi merozoite antigen 1 (EMA1)-based competitive enzyme-linked immunosorbent assay (ELISA)used in the U.S. to detect equine theileriosis detects T. equi but not T. haneyi, and the complexity of molecular assays precludes widespread use for epidemiologic studies. In order to facilitate urgently needed studies on the prevalence of T. haneyi, the goal of this study was to develop a sensitive and specific serologic assay for the diagnosis of T. haneyi based on the equi merozoite antigen 11 (ThEMA11). To achieve this objective, ThEMA11 was recombinantly expressed in eukaryotic cells and its antigenicity assessed using sera from T. haneyi-experimentally infected horses. Confirmation of sera reactivity enabled design and optimization of an indirect ELISA. Specificity of the ELISA for T. haneyi was assessed using a cohort of sera from horses experimentally infected and confirmed PCR-positive for either $T$. equi or T. haneyi. Data from field samples further demonstrate that the ThEMA11 ELISA is capable of identifying T. haneyi antibodies in horses from multiple continents around the world.
\end{abstract}

Keywords: equine theileriosis; Theileria haneyi; enzyme-linked immunosorbent assay (ELISA), serology

\section{Introduction}

Theileria haneyi is an apicomplexan hemoparasite and one of two known causative agents of equine theileriosis. T. haneyi appears to have a global distribution, with infected equids having been identified in North America, South America, and Africa [1-5]. The organism causes milder clinical disease (variable fever, anemia) than T. equi in experimentally infected horses, and is capable of superinfection with T. equi $[3,6]$. Horses remain persistently infected following the acute stage of disease, and these asymptomatic horses are presumed to be reservoirs of infectious organisms for competent tick vectors. Unfortunately, while the antiparasitic drug imidocarb diproprionate (ID) resolves the majority of equine infections with U.S. strains of T. equi, T. haneyi does not appear to be susceptible to ID, and co-infection of horses with T. equi and T. haneyi reduces the efficacy of ID against T. equi [7].

Initial investigation into the serologic immune response to $T$. haneyi revealed that sera from T. haneyi-infected horses react with affinity purified, T. equi (Florida isolate) equi merozoite antigens (EMA) 1 and 2 [6]. Interestingly, genomic analysis revealed that the $T$. haneyi genome lacks the ema1, 3, and 4 genes, but contains three novel EMA family members, designated ema11-13 [3]. Antigenic cross-reactivity is attributed to high amino acid identity within the EMA family, both within the T. equi genome and between the T. equi and T. haneyi genomes $[3,8]$. The EMA family has garnered significant attention in the veterinary diagnostic community, and regulatory T. equi serologic assays approved by The World 
Organization for Animal Health (OIE) and the United States Department of Agriculture (USDA) are based on the equid immune response to the EMAs. The globally validated EMA1-based T. equi competitive enzyme-linked immunosorbent assay (ELISA) detects a wide range of global isolates [9-11]. However, despite the antigenic cross-reactivity observed via immunoblot, the assay does not detect $T$. haneyi infected horses [3,6]. Currently, PCR-based diagnostic assays are the only available means of definitively confirming T. haneyi infection [3,7]. Unfortunately, these molecular diagnostic assays are currently confined to research laboratory use as further optimization is needed to validate the sensitivity of these assays in field-infected animals and to interpret negative results [12].

Due to the aforementioned challenges of molecular assays that currently preclude widespread field use, serology has been the diagnostic assay of choice for detection of infection by the causal agents of equine theileriosis and babesiosis [13]. Therefore, the objective of this study was to develop an indirect ELISA capable of detecting horses infected with T. haneyi. To achieve this objective, EMA11, an EMA protein exclusive to $T$. haneyi (ThEMA11) was selected. Not only would use of this protein allow discrimination between T. equi and T. haneyi infection, since the EMA family is specific to equine Theileria sp. [8], its use allows differentiation between T. haneyi and Babesia caballi infection as well. EMA11 was recombinantly expressed and purified, and sera from known T. haneyiinfected and uninfected horses were utilized for assessment of specific antibody reactivity with recombinant ThEMA11. Confirmation of sera reactivity enabled assay optimization, and indirect ELISA performance was screened against a cohort of horses experimentally infected with either T. equi or T. haneyi to assess specificity. Data from field samples further demonstrate the ThEMA11 indirect ELISA can identify T. haneyi antibodies in horses from multiple continents.

\section{Results}

\subsection{Cloning and Expression of ThEMA11}

The nucleotide sequence of ThEMA11 was codon-optimized for mammalian cell expression, and successfully cloned into pcDNA3.1 (Figure 1a). The recombinant plasmid (pcDNA3.1-ThEMA11) was sequenced to confirm the presence of ThEMA11 in-frame with the cytomegalovirus (CMV) promoter (data not shown). Subsequently, HEK 293t cells were transiently transfected with pcDNA3.1-ThEMA11, and expression of recombinant ThEMA11 was initially confirmed by immunoblot using the anti-6xHis monoclonal antibody (Figure 1b). Results demonstrated the expression of an approximately 33KDa protein, which is the expected molecular weight of ThEMA11 fused with the $6 \times$ His tag. No protein with similar molecular weight was detected in control cells transfected with pcDNA3.1-GFP (Figure 1b). Additional protein bands with molecular weight ranging from 38 to $>80 \mathrm{KDa}$ are also shown in Figure 1b; however, as they are present in both cells transfected with pcDNA3.1 $=$ ThEMA11 or pcDNA3.1-GFP, these are likely non-specific reactions. Once ThEMA11 was successfully expressed, we next sought to evaluate the antigenicity of this recombinant $T$. haneyi protein using serologic assays.

\subsection{Recombinant ThEMA11 Is Recognized by Serum from Thaneyi-Infected Horses, but Not Serum from T. equi-Infected or Uninfected Horses}

After demonstrating expression of ThEMA11 by transiently transfected HEK 293t cells, we next examined whether sera from $T$. haneyi-infected horses recognize the recombinant protein expressed by eukaryotic cells. A crude lysate of the pcDNA3.1-ThEMA11transfected HEK 293t cells was used as antigen for immunoblot analysis. Prior to use in immunoblots, horse sera were adsorbed with HEK 293t-cell lysate to decrease non-specific binding, as described in the Materials and Methods section. Results demonstrated that sera from a horse experimentally infected with $T$. haneyi recognized recombinant ThEMA11 (rec ThEMA11) (Figure 2a). Sera from an uninfected horses did not detect proteins with the expected molecular weight of ThEMA11 (representative uninfected horse serum is shown in Figure 2b). Importantly, sera from T. equi- infected horses did not show cross 
reactivity with ThEMA11 (Figure 2c), despite the high level of amino acid identity between the T. haneyi protein and members of the EMA family in T. equi (Figure S2). The specific recognition of recombinant ThEMA11 by $T$. haneyi-infected horse sera, and the absence of recognition of ThEMA11 by either uninfected or T. equi-infected horse sera prompted us to move forward and use recombinant ThEMA11 to develop an indirect ELISA to detect horse antibodies to T. haneyi.

(a)

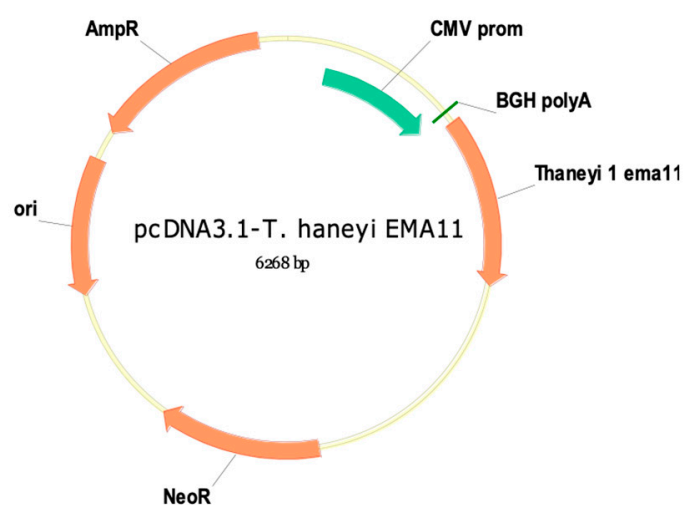

(b)

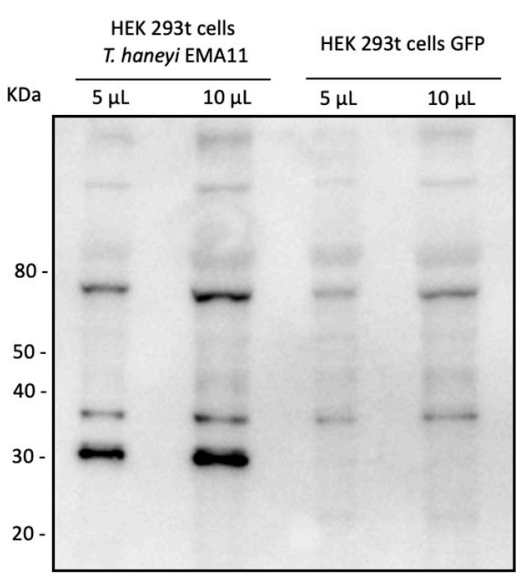

Figure 1. Cloning and expression of T. haneyi EMA11 (ThEMA11). (a) Codon-optimized sequence of ThEMA11 was cloned into pcDNA3.1 in frame with the CMV promoter and fused with the 6 His tag originating the plasmid pcDNA3.1-ThEMA11. (b) Expression of recombinant ThEMA11 in HEK 293t cells detected by anti- $6 \times$ His monoclonal antibody.

(a)

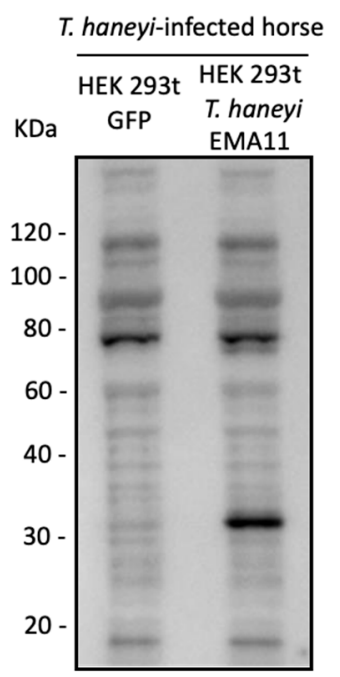

(b)

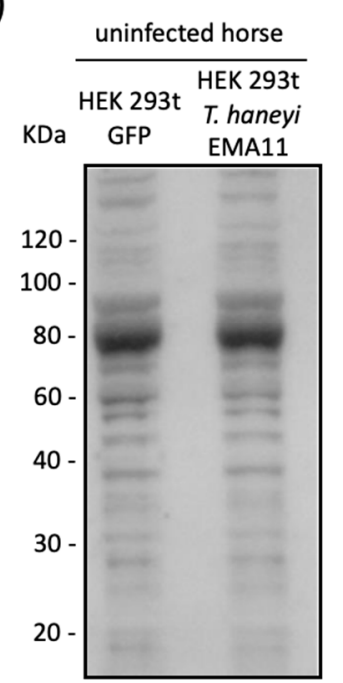

(c)

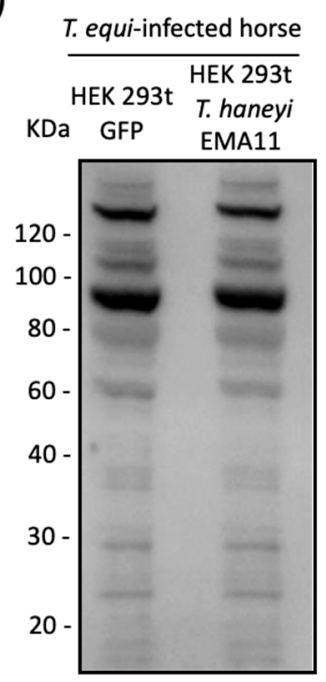

Figure 2. Immunoblot analyses to evaluate the antigenicity of recombinant ThEMA11 using sera from horses experimentally infected with T. haneyi (a), sera from uninfected horses (b), and sera from T. equi infected horses (c). HEK 293t cells expressing GFP were used as a negative control. Blots from one representative animal in each group are shown.

\subsection{Optimization of ThEMA11 as an Antigen for Use in the Indirect ELISA Fformat}

As a first step for ELISA optimization, we scaled up the transient transfection of HEK 293t cells with pcDNA3.1-ThEMA11 and purified the recombinant ThEMA11 using cobalt resin. The level of purity of recombinant ThEMA11 was assessed following each step of the purification process by immunoblot using a monoclonal antibody to the anti-6xHis tag (data not shown). A concentration of approximately $1 \mu \mathrm{g} / \mu \mathrm{L}$ of rec ThEMA11 was obtained 
after purification. Next, the antigenicity of purified ThEMA11 was evaluated using sera from T. haneyi-infected horses (Figure 3). The results of this analysis demonstrate that purified, recombinant ThEMA11 retained its antigenicity and was specifically recognized by sera from $T$. haneyi-infected horses. Immunoblots using sera from two representative, T. haneyi experimentally infected horses, Ho-344 and Ho-777, are shown in Figure 3. Sera from uninfected horses failed to react with purified, recombinant ThEMA11. Immunoblots using sera from two representative uninfected animals, Ho-305 and Ho-404, are presented in Figure 3. Similarly, no reactivity was observed when anti-horse IgG HRP secondary antibodies were used alone (Figure 3).

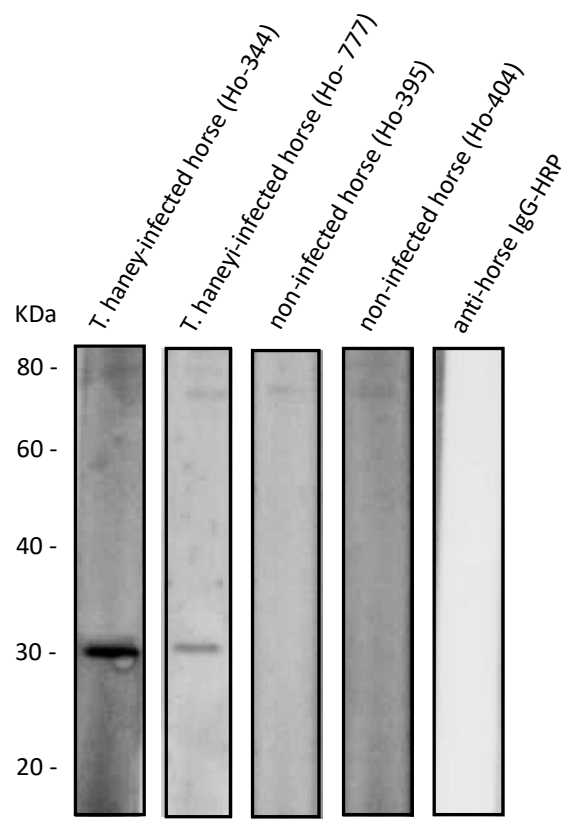

Figure 3. Antigenicity of purified recombinant ThEMA11 using sera from T. haneyi-infected horses (Ho-344 and Ho-777). Sera from uninfected horses (Ho-395 and Ho-404) were used as negative controls. Rabbit anti-horse IgG-HRP alone was also used as a negative control.

Subsequently, the optimal amount of recombinant ThEMA11 was determined in an ELISA format in relation to horse serum dilutions (Figure 4). For this analysis, we used serum from one $T$. haneyi-infected horse and serum from one uninfected horse, selected based on reactivity of the serum with recombinant ThEMA11 in the immunoblots, as described in the previous paragraph. Four serial dilutions of the sera (1:8, 1:16, 1:32, and 1:64), and four different ThEMA11 recombinant protein concentrations ( $5 \mu \mathrm{L} /$ well, $4 \mu \mathrm{L} /$ well, $2 \mu \mathrm{L} /$ well, and $1 \mu \mathrm{L} /$ well) were tested (Figure $4 \mathrm{a}-\mathrm{d}$ ). Data showed that the best discrimination between positive and negative sera was obtained using $4 \mu \mathrm{L} /$ well of antigen with a serum dilution of 1:8 (Figure $4 \mathrm{~b}$ ). Therefore, these parameters were used for the ThEMA11-based ELISA. OD450nm values below 0.2 were obtained when rabbit antihorse IgG HRP was used alone (data not shown). In addition, we used the Rhipicephalus microplus tick antigen Bm86 fused with the 6-His tag (Bm86-6-His) and purified by cobalt column, a similar approach used to purify ThEMA11, to evaluate potential reactivity of horse antibodies to the 6-His tag. No significant reactivity was detected in representative $T$. haneyi-infected and uninfected horse sera against Bm86-6-His, indicating that that 6-His tag is not implicated in the OD signal observed in the ThEMA11 ELISA (data not shown). 
(a)

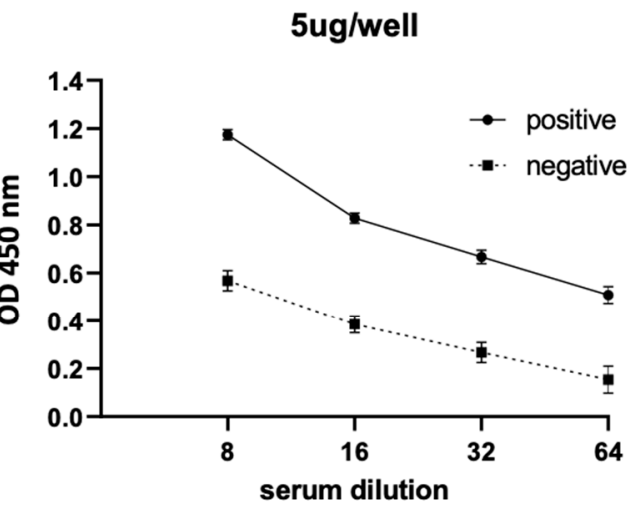

(c)

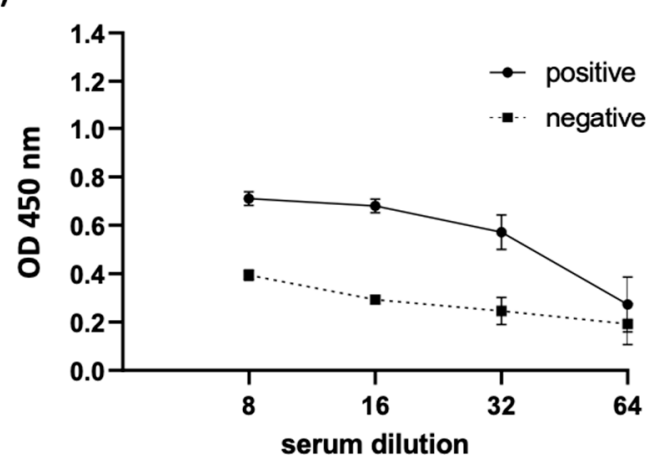

(b)

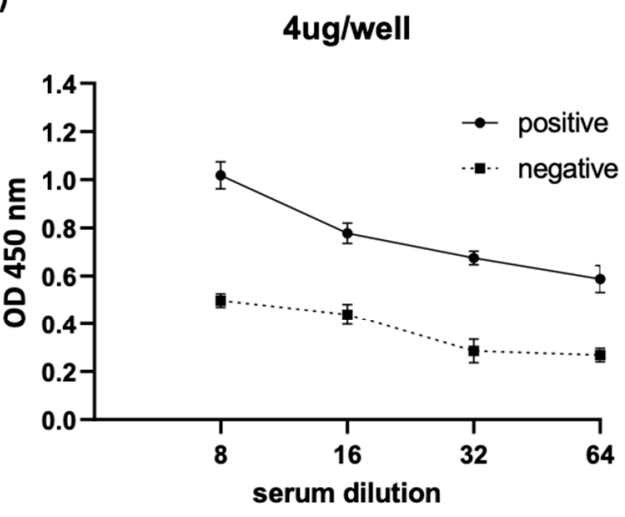

(d)

1ug/well

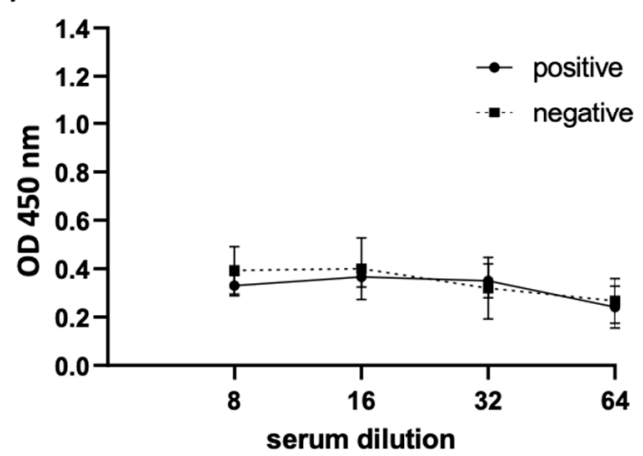

Figure 4. Titration of the optimal amount of recombinant ThEMA11 in an ELISA format in relation to horse serum dilutions. Results show titration of serum from one representative T. haneyi-infected horse and serum from one representative uninfected horse. Four serial dilutions of the sera (1:8, 1:16, 1:32, and 1:64), and four different ThEMA11 recombinant protein concentrations ( $5 \mu \mathrm{L} /$ well, $4 \mu \mathrm{L}$ /well, $2 \mu \mathrm{L}$ /well, and $1 \mu \mathrm{L} /$ well) were tested (a-d). Error bars represent the standard deviation of technical replicates.

A total of 18 serum samples from T. haneyi experimentally infected horses and 19 serum samples from uninfected horses were then used to determine the ThEMA11-based ELISA cutoff (Figure 5). An optimal cutoff of $0.8 \mathrm{OD} 450 \mathrm{~nm}$ was determined, representing the average of negative samples plus three standard deviations. In addition, sera from nine experimentally T. equi-infected, known T. haneyi-negative horses were also analyzed. As demonstrated, sera from all T. equi-infected horses present OD values below the cutoff threshold and therefore, were correctly shown as negative for exposure to T. haneyi (Figure 5). Assessment of 35 field-collected horse serum samples (11 known T. haneyiinfected and 24 known uninfected horses) demonstrated that the recombinant ThEMA11based ELISA correlates well with immunoblot results and showed relative sensitivity and specificity of the new ELISA of $90.90 \%$ and $95.83 \%$, respectively.

\subsection{Use of the ThEMA11-Based ELISA to Detect Antibodies to T. haneyi in Horse Sera from Different Geographic Regions}

After determining the positive threshold, sensitivity, and specificity of the ThEMA11based ELISA, we used the assay to investigate the presence of antibodies against T. haneyi in serum samples from horses residing in or with a travel history to distinct geographical regions (Figure 6). A total of 176 horse sera were tested (145 from U.S., 6 from Germany, 13 from Mexico, 2 from France, 3 from Ireland, 1 from Puerto Rico, 3 from the Netherlands, and three sera from unknown locations). Of the tested samples, 50.56\% $(\mathrm{n}=90)$ were positive for T. haneyi using the ThEMA11 ELISA, and 48.86\% ( $\mathrm{n}=86)$ were considered negative (Figure 6). These equine serum samples were also assessed for T. equi and B. caballi antibodies (Tables 1 and 2). Results demonstrated that 58.62\% (51 serum samples) were 
positive for both T. haneyi EMA11 and T. equi EMA1. In contrast, 62.96\% (51 serum samples) were negative for ThEMA11 but positive for the presence of antibodies to T. equi EMA1. A total of $27.92 \%$ (7 serum samples) were positive both for ThEMA11 and B. caballi RAP-1 antibodies. On the contrary, $29.41 \%$ (5 serum samples) were positive for the presence of B. caballi RAP-1 antibodies, but negative for ThEMA11. Considering the detection of ThEMA11 antibodies in samples that were negative for T. equi EMA1 or B. caballi RAP-1, together the results also reinforce the specificity of the ThEMA11-based ELISA described in this study. Collectively, the results demonstrate the use of the ThEMA11 ELISA to investigate the presence of antibodies to T. haneyi in horse sera from distinct geographical regions around the world.

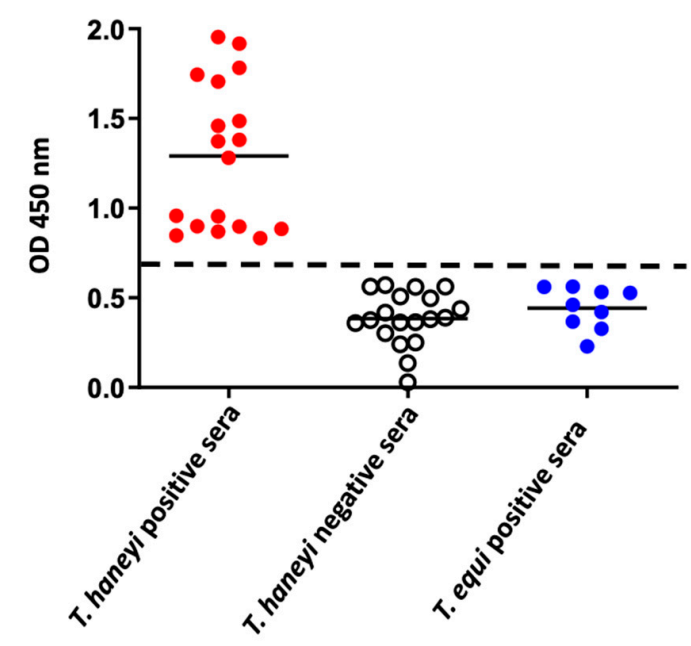

Figure 5. Results of the ThEMA11-based ELISA using serum samples from T. haneyi experimentally infected horses $(n=18)$, from T. equi experimentally infected horses $(n=9)$, and from uninfected horses $(n=19)$. The dashed line indicates the cutoff of 0.8 OD450 $\mathrm{nm}$, representing the average of negative samples (uninfected horse sera) plus three standard deviations. The solid lines represent the average OD for each group.

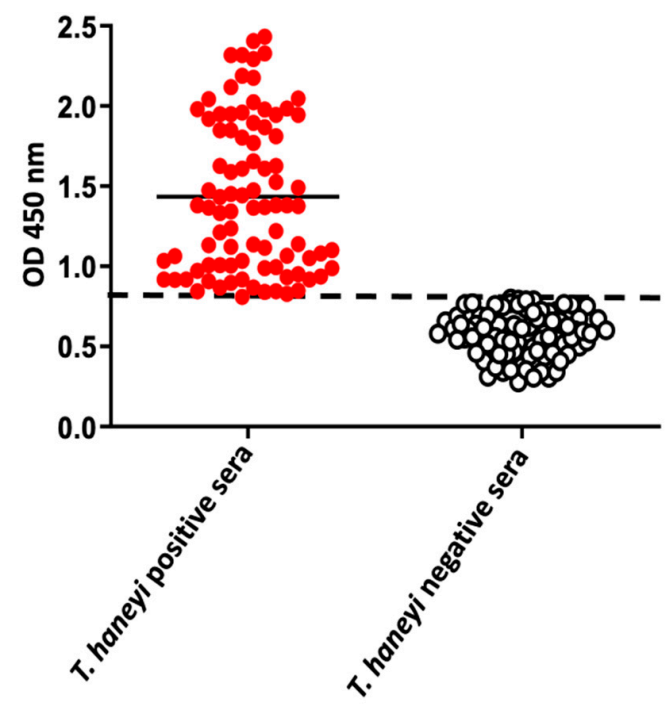

Figure 6. Use of the ThEMA11-based ELISA to investigate the presence of antibodies against T. haneyi in serum samples $(n=176)$ from horses residing in or with a travel history to distinct geographical regions endemic for equine theileriosis. The dashed line indicates the cutoff of $0.8 \mathrm{OD} 450 \mathrm{~nm}$, previously determined representing the average of negative samples (uninfected horse sera) plus three standard deviations. The solid lines represent the average OD for each group. 100/176 equine field serum samples were positive for T. haneyi, and 76/176 were negative. 
Table 1. Presence of T. haneyi EMA11 and T. equi EMA1 antibodies in horse sera from distinct geographic regions.

\begin{tabular}{cccc}
\hline \multicolumn{2}{c}{ T. haneyi Positive Samples $(\mathbf{n}=\mathbf{8 7})$} & \multicolumn{2}{c}{ T. haneyi Negative Samples $(\mathbf{n}=\mathbf{8 1})$} \\
\hline T. equi positive & T. equi negative & T. equi positive & T. equi negative \\
$58.62 \%(\mathrm{n}=51)$ & $41.37 \%(\mathrm{n}=36)$ & $62.96 \%(\mathrm{n}=51)$ & $37.07 \%(\mathrm{n}=30)$ \\
\hline
\end{tabular}

Table 2. Presence of T. haneyi EMA11 and B. caballi RAP-1 antibodies in horse sera from distinct geographic regions.

\begin{tabular}{cccc}
\hline \multicolumn{2}{c}{ T. haneyi Positive Samples $(\mathrm{n}=\mathbf{8 7})$} & \multicolumn{2}{c}{ T. haneyi Negative Samples $(\mathbf{n}=\mathbf{8 1})$} \\
\hline B. caballi positive & B. caballi negative & B. caballi positive & B. caballi negative \\
$26.92 \%(\mathrm{n}=7)$ & $73.07 \%(\mathrm{n}=19)$ & $24.41 \%(\mathrm{n}=5)$ & $70.58 \%(\mathrm{n}=12)$ \\
\hline
\end{tabular}

\section{Discussion}

Currently, the only available diagnostic assay for T. haneyi is nested PCR, which hinders widespread assessment of prevalence of this recently discovered organism. Despite the reported high sensitivity of PCR, especially when utilizing nested PCR, multiple factors can lead to inhibition of reactions, leading to false negative results [14]. Another downside of direct molecular diagnostic assays, such as PCR, is the potential for false-negative results due to low parasitemia in peripheral blood, especially in the chronic phase of $B$. caballi infections. In addition, molecular assays are relatively more expensive. Therefore, serology remains the more widely accepted diagnostic, especially for persistent infections such as those caused by T. equi and T. haneyi.

Since T. equi and T. haneyi are closely related, careful analysis was required to identify a candidate antigen for use in a T. haneyi-specific diagnostic assay. In a previous study, antigenic cross-reactivity was observed to a subset of T. equi proteins in immunoblots, but the T. equi EMA1 based regulatory diagnostic competitive ELISA failed to detect T. haneyi infected horses. For development of a T. haneyi-specific serologic assay, the T. haneyi EMA11 protein was selected because it is exclusive to T. haneyi. The antigen was then carefully evaluated to ensure cross-reactivity did not occur between T. equi and T. haneyi infected horses as there is a high level of amino acid identity between the T. equi and T. haneyi EMA family members. Additionally, a mammalian cell expression system was utilized to ensure proper protein folding, and to reduce bacterial protein contamination and resultant high background reactivity. Following expression and purification, the antigenicity of recombinant ThEMA11 was verified via screening with sera from known infected and noninfected horses. The infection status of these horses was correlated at multiple timepoints with nPCR and/or serial blood smears, and immunoblot using serum from horses in both the acute and chronic stages of infection.

Since serum from horses experimentally infected with only one geographic isolate of $T$. haneyi was used for the initial assay development and optimization steps, we chose to screen a cohort of geographically diverse equine serum samples to gain a broader perspective of the potential use of this assay on a global scale. Performance of the assay using this diverse sample set was strong, and high agreement was noted with the $T$. haneyi immunoblot. Using this information, the serologic assay was determined to have a sensitivity and specificity of $90.9 \%$ and $95.83 \%$, respectively. It was beyond the scope of this study to investigate the level of conservation of ThEMA11 in parasites around the world. However, the fact that the ThEMA11-based ELISA was able to detect antibodies in geographically diverse equine serum samples suggest that EMA11 is highly immunogenic and conserved among worldwide isolates of T. haneyi.

A reliable serologic diagnostic assay for T. haneyi will become crucial for international movement of horses as recently published data suggests that co-infection with $T$. haneyi reduces the efficacy of ID against T. equi [7]. Furthermore, ID lacks efficacy in horses infected with T. haneyi alone [7]. This data is concerning, as potential new anti-Theileria chemotherapeutic compounds are currently only in early testing phase, leaving a paucity of 
potential therapeutic options for infected horses [15-17]. Additionally, although experimental infection with T. haneyi appears to be mild, the clinical signs and severity associated with natural infection remains unknown, and could be more significant, as has been documented with T. equi.

The assay developed in this study will enable subsequent, global evaluation of the prevalence of T. haneyi. Following more extensive validation, use of this assay in screening horses prior to inter-country movement may help with the continued control on equine theileriosis on a global scale.

\section{Materials and Methods}

\subsection{T. haneyi EMA11 Cloning, Expression, and Purification}

T. haneyi parasites and the ThEMA11 sequence used in this study were previously described in $[3,8]$. The full-length sequence of ThEMA11 was codon-optimized for mammalian cells, and a $6 \mathrm{His}$ tag added to its C-terminal end (GeneArt, Thermo Fisher Scientific, Waltham, MA, USA) (Figure S1). The synthetic gene was then cloned into pcDNA3.1 and the plasmid, termed pcDNA3.1-ThEMA11, was then used to express the target protein in human embryonic kidney (HEK) 293t cells $\left(\right.$ ATCC $^{\circledR}$, Gaithersburg, MD, USA).

For the expression of recombinant ThEMA11, HEK 293t cells were transiently transfected with pcDNA3.1-ThEMA11 using polyethylenimine (PEI) transfection reagent per standard protocols. Briefly, HEK 293t cells were seeded overnight in six-well plates (70 to $80 \%$ confluence) and transfected with a target plasmid using PEI ( $1 \mu$ g plasmid DNA: $4 \mu \mathrm{L}$ PEI ratio), as previously described [18]. Four hours after transfection, the transfection mix was replaced with complete Dulbecco's modified essential medium (cDMEM) (10\% fetal bovine serum, $24 \mathrm{mM}$ of HEPES, $2 \mathrm{mM}$ of L-glutamine, $100 \mathrm{IU} / \mathrm{mL}$ penicillin, and $100 \mu \mathrm{g} / \mathrm{mL}$ streptomycin). $48 \mathrm{~h}$ after transfection, cells were collected in $1 \times$ Cell Culture Lysis Reagent (Promega, Madison, WI, USA) containing the Halt Protease Inhibitor Single Use Cocktail, EDTA-Free (Thermo Fisher Scientific). Lysate of transfected HEK 293t cells were stored at $-80{ }^{\circ} \mathrm{C}$ until use for immunoblot analysis and protein purification.

Recombinant ThEMA11 was purified using the HisPur ${ }^{\mathrm{TM}}$ Cobalt Purification Kit following the manufacturer's protocol (Thermo Fisher Scientific). Briefly, transient transfection of HEK 293t was scaled up to be performed in $150 \mathrm{~cm}^{2}$ cell culture flasks. Forty-eight hours post-transfection, the culture supernatant was removed, and cells re-suspended in $1 \times$ PBS containing Halt Protease Inhibitor Single Use Cocktail, EDTA-Free. Cells were then lysed in three freeze-thaw cycles and stored at $-80^{\circ} \mathrm{C}$ until purification. For protein purification, cobalt columns were equilibrated to room temperature and washed in Equilibration/Wash Buffer provided by the HisPur ${ }^{\mathrm{TM}}$ purification kit. The lysate of pcDNA3.1-ThEMA11-transfected HEK 293t cells was loaded into the columns and incubated for $30 \mathrm{~min}$ at $4^{\circ} \mathrm{C}$ on a rocking platform. Columns were then centrifuged at $700 \times g$ for $2 \mathrm{~min}$, and the resin washed twice in Equilibration/Wash Buffer. Target protein was eluted by adding one resin-bed volume of Elution Buffer provided by the HisPur ${ }^{\mathrm{TM}}$ purification kit followed by centrifugation at $700 \times g$ for $2 \mathrm{~min}$. Elution steps were repeated twice, and fractions were stored at $-80^{\circ} \mathrm{C}$ until use.

\subsection{Horse Serum Samples}

Serum samples from T. haneyi experimentally infected horses $(n=13)[3,6,7]$ were collected at multiple time points following infection and used as positive controls. Serum samples ( $\mathrm{n}=19$ ) from healthy horses located in WA or ID, USA (where T. equi and T. haneyi are not naturally present in equine populations), which were confirmed negative for $T$. haneyi via $\mathrm{nPCR}$ and blood smear cytology were used as negative controls in this study. The known T. haneyi-positive and negative sera were used to determine the ThEMA11-based ELISA cutoff. In addition, serum samples from confirmed T. equi-infected horses $(\mathrm{n}=8)$ were also used to assess the specificity of the T. haneyi EMA11-based ELISA. All serum samples were obtained from horses housed at the USDA-ADRU, University of Idaho, or Washington State University animal facilities. All animal experiments were approved by 
the Washington State University and University of Idaho Institutional Animal Care and Use Committees, ASAF numbers 4973 and 6241 (Washington State University, Pullman, WA, USA) and 2016-18 and 2016-28 (University of Idaho, Moscow, ID, USA).

After establishing the basic positive/negative cutoff and ensuring that T. equi infected horse serum did not recognize EMA11, as described above, field horse serum samples $(n=176)$ from different regions around the world were used to begin testing the assay in a diverse sample set. The serum samples were from horses in the U.S., Mexico, and Germany, among other countries. Serum samples were obtained through collaboration with OIE Reference Laboratory for equine piroplasmosis located at Washington State University. Reactivity of these samples for T. equi (168/176) and/or B. caballi (43/176), depending on the individual case circumstances, was determined by the Reference Laboratory via commercially available cELISAs and immunoblots as described below. Due to individual horse circumstances, not all samples were assayed for both pathogens (some were tested for just T. equi, some for just B. caballi, and some for both pathogens). The date of collection and sample source location are presented in Table S1.

\subsection{Competitive ELISA (cELISA) for T. equi or B. caballi}

Horse sera tested in this study for the presence of anti-T. haneyi antibodies were evaluated by cELISA to detect antibodies to T. equi and/or B. caballi as previously described in Knowles et al 1992 and Kappmeyer et al 1999, respectively [19,20].

\subsection{Immunoblot Analysis}

Immunoblots were performed using lysates of HEK 293t transfected with pcDNA3.1ThEMA11 or with purified recombinant ThEMA11 as antigen and sera from known $T$. haneyi-infected horses or monoclonal antibody $(\mathrm{mAb})$ recognizing the $6 \mathrm{His}$ tag (clone AD1.1.10; Bio-Rad, Hercules, CA, USA). In addition, sera from healthy, uninfected horses and confirmed T. equi-infected horses were used as controls in the immunoblots. Briefly, 5 or $10 \mu \mathrm{L}$ of cell lysate or $5 \mu \mathrm{L}$ of recombinant purified ThEMA11 was loaded into each lane and separated in NuPAGE ${ }^{\mathrm{TM}} 4-12 \%$ Bis-Tris gel (Invitrogen, Waltham, MA USA). The gel was then electrotransferred to a nitrocellulose membrane and blocked with PBS 0.02\% Tween 20 (PBS-T) 10\% non-fat milk. After blocking, the membrane was incubated for $1 \mathrm{~h}$ at room temperature with either horse serum (1:50 or 1:100) or the anti-6His tag mAb (1:500). For immunoblots using lysate of HEK 293t cells transfected with pcDNA3.1ThEMA11, horse sera were adsorbed as follows. Serum samples were incubated for $48 \mathrm{~h}$ at $4{ }^{\circ} \mathrm{C}$ with lysate of wildtype HEK 293 cells (approximately $10^{6}$ cells). After that, the mix was centrifuged $(1000 \times g, 5 \mathrm{~min})$ and supernatant used for the immunoblots. After washing in PBS-T, the membrane was incubated for $1 \mathrm{~h}$ at room temperature with secondary anti-mouse IgG HRP (SeraCare; 1:2500) or anti-horse IgG HRP (SeraCare; 1:2500). The immune complexes were revealed using an enhanced chemiluminescence method (ECL ${ }^{\mathrm{TM}}$; Amersham, Buckinghamshire, UK).

\subsection{Indirect ELISA}

Immunlon ${ }^{\mathrm{TM}} 2 \mathrm{HB}$ 96-well microtiter ELISA plates (Thermo Scientific, Hanover Park, IL, USA) were coated overnight at $4{ }^{\circ} \mathrm{C}$ with $50 \mu \mathrm{L}$ of recombinant purified ThEMA11 ( $4 \mu \mathrm{g} /$ well) in $1 \times$ Coating Buffer (BioLegend, San Diego, CA, USA). After that, excess antigen was removed, and the plates were blocked with $200 \mu \mathrm{L} /$ well of Blocker ${ }^{\mathrm{TM}}$ Casein in PBS (Thermo Fisher Scientific) at room temperature (RT) for $1 \mathrm{~h}$. Following the blocking step, serial dilutions (1:2 to 1:256) of T. haneyi-positive or negative horse sera were added to the plates and incubated at RT for $1 \mathrm{~h}$. After five washes in $0.05 \%(v / v)$ Tween-20 in PBS (PBS-T), rabbit anti-horse IgG-HRP (1:10,000) (Millipore-Sigma, St. Louis, MO, USA) was added to each well, and the plates were incubated at RT for $1 \mathrm{~h}$. Plates were then washed five times in PBS-T and developed with $100 \mu \mathrm{L}$ of 1 -Step ${ }^{\mathrm{TM}}$ Ultra TMB-ELISA Substrate Solution (Thermo Fisher Scientifics). The enzymatic reaction was stopped via addition of $100 \mu \mathrm{L}$ of TMB Stop Solution (0.2 $\left.\mathrm{M} \mathrm{H}_{2} \mathrm{SO}_{4}\right)$ (SeraCare, Gaithersburg, MD, 
USA) to each well, and plates read at $450 \mathrm{~nm}$ using an ELISA plate reader (MultiSkan MCC, Thermo Fisher Scientific). Immunoblot was used as gold standard to evaluate sensitivity and specificity of the ThEMA11-based ELISA. Sensitivity and specificity were calculated using the following formula: sensitivity $=$ [number of true positive samples - number of false negative samples) $\times 100] /$ number of true positive samples; and specificity $=$ [number of true negative samples - number of false positive samples) $\times 100] /$ number of true negative samples.

Supplementary Materials: The following are available online at https:/ / www.mdpi.com/2076-0 817/10/3/270/s1, Figure S1: Codon-optimized Theileira haneyi EMA11 for mammalian expression. Figure S2: Amino acid alignment of Theileira haneyi EMA11 and T. equi EMA1. Table S1: List of field horse samples.

Author Contributions: Conceptualization, L.M.F., R.G.B., M.W.U., and D.P.K.; methodology, L.M.F., R.G.B., L.K., M.W.U., and D.P.K.; formal analysis, L.M.F., R.G.B., L.K., D.P.K.; investigation, R.G.B., K.P.S., L.K., and K.D.D.; resources, L.M.F., M.W.U., and D.P.K.; data curation, R.G.B.; writing—original draft preparation, R.G.B., K.P.S., and L.M.F.; writing-review and editing, L.K., M.W.U., D.P.K. and K.D.D.; visualization, R.G.B.; supervision, L.M.F., M.W.U., and D.P.K.; project administration, L.M.F., D.P.K. and M.W.U.; funding acquisition, D.P.K., L.M.F., and M.W.U. All authors have read and agreed to the published version of the manuscript.

Funding: The research was funded by USDA-ARS CRIS\# \#2090-320000-039-00D and in part by the Washington State University, College of Veterinary Medicine Equine Infectious Disease Research Program.

Institutional Review Board Statement: All animal experiments were approved by the Washington State University and University of Idaho Institutional Animal Care and Use Committees, ASAF numbers 4973 and 6241 (Washington State University) and 2016-18 and 2016-28 (University of Idaho).

Informed Consent Statement: Not applicable.

Data Availability Statement: Data is contained within the article and supplementary material. Additional raw data is available on request from the corresponding author.

Acknowledgments: The authors wish to acknowledge the technical expertise of Shelby Beckner, and the animal handling expertise of Cody Evans, Morgan Burke, Emma Karel, Megan Jacks, and Ralph Horn.

Conflicts of Interest: The authors declare no conflict of interest. The funders had no role in the design of the study; in the collection, analyses, or interpretation of data; in the writing of the manuscript, or in the decision to publish the results.

\section{References}

1. Bishop, R.P.; Kappmeyer, L.S.; Onzere, C.K.; Odongo, D.O.; Githaka, N.; Sears, K.P.; Knowles, D.P.; Fry, L.M. Equid infective Theileria cluster in distinct $18 \mathrm{~S}$ rRNA gene clades comprising multiple taxa with unusually broad mammalian host ranges. Parasit Vectors 2020, 13, 261. [CrossRef] [PubMed]

2. $\quad$ Bhoora, R.V.; Collins, N.E.; Schnittger, L.; Troskie, C.; Marumo, R.; Labuschagne, K.; Smith, R.M.; Dalton, D.L.; Mbizeni, S. Molecular genotyping and epidemiology of equine piroplasmids in South Africa. Ticks Tick Borne Dis. 2019, 11, 101358. [CrossRef] [PubMed]

3. Knowles, D.P.; Kappmeyer, L.S.; Haney, D.; Herndon, D.R.; Fry, L.M.; Munro, J.B.; Sears, K.; Ueti, M.W.; Wise, L.N.; Silva, M.; et al. Discovery of a novel species, Theileria haneyi n. sp., infective to equids, highlights exceptional genomic diversity within the genus Theileria: Implications for apicomplexan parasite surveillance. Int. J. Parasitol. 2018, 48, 679-690. [CrossRef] [PubMed]

4. Vieira, M.I.B.; Costa, M.M.; de Oliveira, M.T.; Goncalves, L.R.; Andre, M.R.; Machado, R.Z. Serological detection and molecular characterization of piroplasmids in equids in Brazil. Acta Trop. 2018, 179, 81-87. [CrossRef] [PubMed]

5. Mshelia, P.W.; Kappmeyer, L.; Johnson, W.C.; Kudi, C.A.; Oluyinka, O.O.; Balogun, E.O.; Richard, E.E.; Onoja, E.; Sears, K.P.; Ueti, M.W. Molecular detection of Theileria species and Babesia caballi from horses in Nigeria. Parasitol. Res. 2020, 119, $2955-2963$. [CrossRef] [PubMed]

6. $\quad$ Sears, K.P.; Kappmeyer, L.S.; Wise, L.N.; Silva, M.; Ueti, M.W.; White, S.; Reif, K.E.; Knowles, D.P. Infection dynamics of Theileria equi and Theileria haneyi, a newly discovered apicomplexan of the horse. Vet. Parasitol. 2019, 271, 68-75. [CrossRef] [PubMed]

7. Sears, K.; Knowles, D.; Dinkel, K.; Mshelia, P.W.; Onzere, C.; Silva, M.; Fry, L. Imidocarb Dipropionate Lacks Efficacy against Theileria haneyi and Fails to Consistently Clear Theileria equi in Horses Co-Infected with T. haneyi. Pathogens 2020, 9, 1035. [CrossRef] [PubMed] 
8. Wise, L.N.; Kappmeyer, L.S.; Knowles, D.P.; White, S.N. Evolution and diversity of the EMA families of the divergent equid parasites, Theileria equi and T. haneyi. Infect. Genet. Evol. 2019, 68, 153-160. [CrossRef]

9. Knowles, D.P., Jr.; Perryman, L.E.; Goff, W.L.; Miller, C.D.; Harrington, R.D.; Gorham, J.R. A monoclonal antibody defines a geographically conserved surface protein epitope of Babesia equi merozoites. Infect. Immun. 1991, 59, 2412-2417. [CrossRef]

10. Cunha, C.W.; Kappmeyer, L.S.; McGuire, T.C.; Dellagostin, O.A.; Knowles, D.P. Conformational Dependence and Conservation of an Immunodominant Epitope within the Babesia equi Erythrocyte-Stage Surface Protein Equi Merozoite Antigen 1. Clin. Vaccine Immunol. 2002, 9, 1301-1306. [CrossRef]

11. Ueti, M.W.; Palmer, G.H.; Kappmeyer, L.S.; Scoles, G.A.; Knowles, D.P. Expression of Equi Merozoite Antigen 2 during Development of Babesia equi in the Midgut and Salivary Gland of the Vector Tick Boophilus microplus. J. Clin. Microbiol. 2003, 41, 5803-5809. [CrossRef] [PubMed]

12. Wise, L.N.; Kappmeyer, L.S.; Silva, M.G.; White, S.N.; Grause, J.F.; Knowles, D.P. Verification of post-chemotherapeutic clearance of Theileria equi through concordance of nested PCR and immunoblot. Ticks Tick Borne Dis. 2018, 9, 135-140. [CrossRef] [PubMed]

13. Wise, L.N.; Kappmeyer, L.S.; Mealey, R.H.; Knowles, D.P. Review of equine piroplasmosis. J. Vet. Intern. Med. 2013, 27, 1334-1346. [CrossRef]

14. Schrader, C.; Schielke, A.; Ellerbroek, L.; Johne, R. PCR inhibitors-Occurrence, properties and removal. J. Appl. Microbiol. 2012, 113, 1014-1026. [CrossRef] [PubMed]

15. Silva, M.G.; Villarino, N.F.; Knowles, D.P.; Suarez, C.E. Assessment of Draxxin ${ }^{\circledR}$ (tulathromycin) as an inhibitor of in vitro growth of Babesia bovis, Babesia bigemina and Theileria equi. Int. J. Parasitol. Drugs Drug Resist. 2018, 8, 265-270. [CrossRef] [PubMed]

16. Bowden, G.D.; Reis, P.M.; Rogers, M.B.; Bone Relat, R.M.; Brayton, K.A.; Wilson, S.K.; Di Genova, B.M.; Knoll, L.J.; Nepveux, V.F.; Tai, A.K.; et al. A conserved coccidian gene is involved in Toxoplasma sensitivity to the anti-apicomplexan compound, tartrolon E. Int. J. Parasitol. Drugs Drug Resist. 2020, 14, 1-7. [CrossRef] [PubMed]

17. O'Connor, R.M.; Nepveux, V.F.; Abenoja, J.; Bowden, G.; Reis, P.; Beaushaw, J.; Bone Relat, R.M.; Driskell, I.; Gimenez, F.; Riggs, M.W.; et al. A symbiotic bacterium of shipworms produces a compound with broad spectrum anti-apicomplexan activity. PLoS Pathog. 2020, 16, e1008600. [CrossRef] [PubMed]

18. Bastos, R.G.; Sears, K.; Dinkel, K.D.; Knowles, D.P.; Fry, L.M. Changes in the Molecular and Functional Phenotype of Bovine Monocytes during Theileria parva Infection. Infect. Immun. 2019, 87. [CrossRef] [PubMed]

19. Knowles, D.P.; Kappmeyer, L.S.; Stiller, D.; Hennager, S.G.; Perryman, L.E. Antibody to a Recombinant Merozoite Protein Epitope Identifies Horses Infected with Babesia equi. J. Clin. Microbiol. 1992, 30, 3122-3126. [CrossRef] [PubMed]

20. Kappmeyer, L.S.; Perryman, L.E.; Hines, S.A.; Baszler, T.V.; Katz, J.B.; Hennager, S.G.; Knowles, D.P. Detection of equine antibodies to Babesia caballi by recombinant B. caballi rhoptry-associated protein 1 in a competitive-inhibition enzyme-linked immunosorbent assay. J. Clin. Microbiol. 1999, 37, 2285-2290. [CrossRef] [PubMed] 